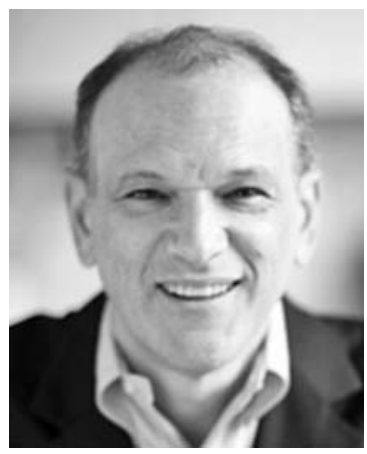

\title{
A Conversation with Eric Nestler
}

\author{
INTERVIEWER: JAN WITKOWSKI \\ Executive Director of the Banbury Center \\ at Cold Spring Harbor Laboratory
}

Dr. Nestler is a Nash Family Professor and Chair of the Department of Neuroscience, and Director of the Friedman Brain Institute, at Mount Sinai, and currently a member of the National Advisory Drug Abuse Council.

Jan Witkowski: One of the problems about using mice to study higher level brain functions is finding a model that to some degree reflects what one might expect in humans.

Dr. Nestler: Yes, exactly. Most of the depression field has utilized what are called despair tests, something like the forced swim test where a mouse or rat would be placed in a beaker of water. It would swim for a while, then stop. People say that when it stops it's despairing although we really have no reason to think that. When you give the animal an acute dose of an antidepressant, it swims a little longer. It's hard to imagine a test with so little validity being so widely used in depression research.

Several years ago we set out to try to develop a test that is better validated and has more ethological relevance for a mouse. We figured that social stress was a domain of behavior that really hadn't been studied very much in animal depression models. In this model, which we call social defeat, a normal inbred C57 mouse is placed in the cage of a retired male breeder CD1 mouse. The retired breeder is bigger and meaner and as soon as the test mouse is placed in that cage, the larger mouse attacks it.

We only allow the physical interaction to occur for a couple of minutes before placing a screen between the mice. The test mouse continues to be subjected to all the aggressive cues of the larger mouse but has no more physical contact for the rest of the day. We repeat that process every day, typically for 10 days. Afterwards, we can show that we have induced, in that C57 mouse, a behavioral syndrome that's characterized by anhedonia, which means a loss of interest in pleasurable activities. The mice are less interested in drinking sugar water, eating Reese's Pieces, having sex, or running on a running wheel.

Dr. Nestler: The mice also show anxiety-like behaviors. They develop a metabolic syndrome: they eat too much and get fat and develop a prediabetic state. And they become profoundly socially avoidant. You might think that, after 10 days of being beaten up by different mice, social avoidance is a normal response, but we've shown if these defeated mice are put back in normal caging with the brothers with whom they were raised, for the rest of their lives they remain avoidant and don't play in the cages like their brothers. So we think this behavior is clearly pathologic.

The behaviors are corrected by chronic (not acute) administration of antidepressants that work in human. Interestingly, there's a small subset of C57 mice that don't develop the syndrome and we call them resilient. They've been subjected to the same level of stress yet somehow they manage to avoid most of these deleterious changes.

Jan Witkowski: Only males respond this way?

Dr. Nestler: This is only a male test. We are very interested in adapting social defeat for females but it's something that's still in progress.

Jan Witkowski: Do you know anything about the difference between the resilient and the susceptible mice?

Dr. Nestler: Right now we have no measure that we can apply to a C57 mouse before the stress that predicts accurately whether they're going to be susceptible or resilient, so it's a latent feature in these animals. After the fact, we have described very large differences in brain and peripheral measures that distinguish resilient and susceptible animals and seem to drive those different states but we don't know what initiates them.

Jan Witkowski: How did you get interested in chromatin remodeling in relation to this induced phenotype?

Dr. Nestler: Over the years my lab has been interested in mechanisms by which an environmental stimulus, a drug of abuse, a stress, might produce long-lasting changes. Because we know in humans that, in addiction syndromes or depression syndromes, environmental experiences can change people profoundly for their lifetimes and make them more or less vulnerable to the condition involved. We've thus been struggling to identify the ways in which an environmental stimulus can change the brain permanently. About 10 years ago studies in developmental biology and cancer biology showed that epigenetic 
modifications of DNA and histones, and other mechanisms, can cause lifelong changes. We basically applied that insight to the brain, asking whether epigenetic modifications could mediate similarly lifelong changes in brain and behavior.

Jan Witkowski: Did you do a screen for chromatin or epigenetically related molecules?

Dr. Nestler: Yes. Over the last five years we've screened in many different ways. We've screened different histone modifications and different chromatin remodeling proteins. Chromatin remodelers are ATPase-containing complexes that control the movement of nucleosomes along a strand of DNA. We were particularly struck that, when we carried out RNA-Seq to look at global changes in RNA expression in different brain areas that occur as a consequence of susceptibility or resilience, the genes encoding specific types of chromatin remodeling proteins were among the most highly regulated. So in the last couple of years we have focused on this family of proteins.

Jan Witkowski: In particular Acf1?

Dr. Nestler: Yes. Acf1 is an accessory subunit of the ISWI family of chromatin remodeling proteins. There are several families. ISWI includes a critical subunit called Snf2h. As an ATPase, Snf2h can bind to certain subunits and drive mechanisms that activate transcription or bind to other subunits like Acf1 and form complexes that are repressive and decrease gene expression.

Jan Witkowski: They're repressed by altering chromatin structure?

Dr. Nestler: That's right. These complexes repress gene expression by redistributing nucleosomes along a strand of DNA, condensing them and filling in nucleosomes in certain regions. When a gene is active, its promoter region is relatively spared of nucleosomes. Acf1-Snf2h inhibitory complexes would basically fill in nucleosomes in that region and suppress that gene.

Jan Witkowski: The question is, what did you find when you looked in resilient animals? I guess you used the resistant ones as a second type of control?

Dr. Nestler: Yes. We always look at the resilient animals since they provide an interesting counterpoint to how an animal adapts or responds to stress. What we found is that, in a brain area called the nucleus accumbens, which is one of the brain's reward regions, induction of Acfl and induction of the Acf1-Snf2h complex occurs only in susceptible animals, not in resilient animals, suggesting that the induction may be a factor in causing depression.

Importantly when we look at this nucleus accumbens brain area in human postmortem brain tissue, we see induction of Acf1 in depressed individuals. This is after death but it suggests that the findings in mice are relevant to the human situation.

Jan Witkowski: So now that you've found this difference in the chromatin remodeling between susceptible and resilient mice, what genes is it working on?
Dr. Nestler: The first step is to show directly that the Acf1Snf $2 \mathrm{~h}$ complex is involved functionally in mediating susceptibility. Using viral vectors, we were able to overexpress Acf1 or knock it down specifically in the nucleus accumbens of an adult mouse. We found that when we overexpressed Acf1 we made the mice more susceptible, and when we knocked down Acf1, preventing the effect seen in susceptibility, we made the mice more resilient.

Dr. Nestler: Then we used chromatin immunoprecipitation sequencing to look genome wide at all the genes that in susceptible mice show increased binding of the Acf1Snf $2 \mathrm{~h}$ inhibitory complex in nucleus accumbens. We've identified a dozen or so interesting genes. They've never been implicated before in stress responses or depression, so the next step is to determine if these genes act alone or in concert with one another to mediate susceptibility.

Jan Witkowski: Will you do that, again, by overexpression and knockdown?

Dr. Nestler: Yes. We'll use the viral vectors initially and then mutant mice as they are available and explore how manipulating the activity or levels of these genes in the nucleus accumbens controls resilience versus susceptibility.

Jan Witkowski: What are the genetics of acute or severe depression in human beings?

Dr. Nestler: Depression is considered moderately heritable, with about a $40 \%$ heritability rate, but it's been very difficult to identify individual genes involved. Accordingly, there is a high discordance among identical twins as well. There is only about a $40 \%$ chance that if one identical twin is depressed, his or her identical twin will also be depressed.

Jan Witkowski: So even for identical twins growing up in the same environment, there may be environmental influences, maybe after they left home.

Dr. Nestler: People have talked about difference in position in the uterus and birth factors and things like that.

Jan Witkowski: Right. But your mice are growing up in a controlled environment.

Dr. Nestler: Right. We are using genetically identical animals and we try to raise them in as identical a manner as possible, yet we see this very large divergence between a susceptible response and a resilient response.

Jan Witkowski: The classic definition of epigenetic is a transgenerational change in gene expression that doesn't involve structural changes in DNA. Have you been able to demonstrate such a phenomenon?

Dr. Nestler: In an experiment in my lab, male mice that had been subjected to social defeat stress were bred to normal females. The males were removed as soon as the females were impregnated, and the mothers were allowed to raise their pups. Then we studied the offspring when they were adults. What we found was really quite dramatic. The offspring of defeated fathers demonstrated much greater 
susceptibility to stress. They had an anxiety-like phenotype at baseline and were much more susceptible to chronic social stress. That was an astounding finding, which gets us into the area of Lamarckian mechanisms that supposedly have been disproved for a century or more.

Jan Witkowski: Have you looked at Acf or the genes affected by Acf1 in the offspring before you stress them?
Dr. Nestler: Not yet. It would be hard to do that before the stress because we have to kill the animal to analyze the state of its genes in brain. However, if we knew that the sons of defeated fathers, for example, showed this increased inherent vulnerability, we could simply analyze their brains and see what changes exist there. It's something we haven't done yet but are keen to do. 


\section{$\$_{\text {CSH\& }}^{\infty}$ Cold Spring Harbor Symposia SYMPOSIA On Quantitative Biology}

\section{A Conversation with Eric Nestler}

Cold Spring Harb Symp Quant Biol 2014 79: 282-284

Access the most recent version at doi:10.1101/sqb.2014.79.13

\section{License}

Email Alerting Receive free email alerts when new articles cite this article - sign up in Service the box at the top right corner of the article or click here. 